\title{
Lifestyle Factors Contributing to HPA-Axis Activation and Chronic Illness in Americans
}

\author{
Dawn Elise Snipes* \\ PhD, LPC-MHSP, LMHC Executive Director, AllCEUs.com, USA
}

*Corresponding author: Dawn-Elise Snipes, PhD, LPC-MHSP, LMHC Executive

Director, AllCEUs.com, USA.

Received Date: October 26, 2019

Published Date: October 31, 2019

\section{Introduction}

More than $50 \%$ of Americans suffer from one or more chronic conditions with an estimated cost of $\$ 3.3$ trillion annually including major depressive disorder (20\%), generalized anxiety disorder (18.1\%), low testosterone (25\%) estrogen imbalances, diabetes (9.2\%), hypertension, autoimmune disorders (23\%), chronic pain, metabolic syndrome (30\%),cardiovascular disease (44\%) and hypothyroid (4.6\%), all of which are associated with disturbances of the hypothalamic-pituitary-adrenal (HPA)-Axis. (National Center for Chronic Disease Prevention and Health Promotion 2019 [1-6].

\section{The HPA-Axis}

The hypothalamic-pituitary-adrenal (HPA)-axis is a key system involved in physiological homeostasis. [7]. It modulates and is modulated by a variety of inhibitory and excitatory systems. Some level of activation of the HPA-Axis is necessary for motivation and energy. When the HPA axis is activated in response to stress it impacts the balance of the neurotransmitters dopamine, serotonin, norepinephrine, GABA, and glutamate; modulates the release of inflammatory cytokines, estrogen and testosterone; and impacts insulin sensitivity and the balance of T3:T4 thyroid hormones. Sustained activation of this bidirectional system results in brain changes which alter hormones and monoamines (neurotransmitters) leading to further HPA-Axis activation and dysrergulation. [8].

When exposed to a physical, environmental or social stressor, the HPA-Axis is activated and prompts the "fight or flight" reaction [6]. Neurons in the paraventricular nucleus (PVN) of the hypothalamus release corticotropin releasing factor (CRF) and arginine vasopressin (AVP) to stimulate the anterior pituitary gland to produce and secrete adrenocorticotropic hormone (ACTH). ACTH causes glucocorticoid (cortisol) synthesis and release from the adrenal glands as well as the release of activation of proinflammatory cytokines (IL-1, IL-4, IL-6, IL-18, and TNF- $\alpha$ ) [9].

Cortisol's primary function is to increase blood glucose and modify fat and protein metabolism to fuel the fight or flight reaction and modulate immune and brain function to help the person effectively manage stressors [8]. It initially impacts the immune system and causes a potent anti-inflammatory response which allows the organism to react to the stressor without being hampered by the pain or fatigue. As cues of the threat wane, the body increases inflammation by releasing proinflammatory cytokines interleukin-1B IL-1, interleukin-6 (IL-6), and TNF- $\alpha$, to accelerate wound healing caused by possible injury $[6,10]$.

When compared to positive events, negative events, or "stress" causes greater awareness and recall of event details which leads to stronger encoding of negative or stressful events than pleasant events. According to the NEVER (Negative Emotional Valence Enhances Recapitulation) model of emotional valence, the greater the number of stimuli related to the unpleasant event that are remembered, the greater the likelihood that the person will encounter reminders of the event leading to increased recapituation. This serves a survival function since there are more significant consequences to responding inappropriately or not protecting oneself from negative event. According to Dr. Aaron BenZeev, people tend to perseverate on negative information and events five times more than positive ones $[11,12]$. Recapitulation initially leads to repeated HPA-Axis activation, but over time the continued stress prolongs the inflammatory response via continued activation of the HPA-Axis leading to glucocorticoid resistance causing cells to become less sensitive to cortisol to protect them from the persistent secretion. This is referred to as hypocortisolism.

Hypocortisolism allows for elevations in systemic inflammation to occur together with increases in cortisol. This prolonged 
inflammation is associated with chronic conditions including arthritis, diabetes, obesity, heart disease, certain cancers, and Alzheimer's disease [6]. Other physiological changes that occur as a result of gluticorticoid resistance or hypocortisolism include...

- Disinhibition of corticotropin releasing hormone (CRH) and norepinephrine which lead to an exaggerated response to acute stressors and corresponding increase in cortisol [13].

- Exaggerated elevation of cortisol during exposure to acute stressors increases the sensitivity of NMDA receptors, which makes the brain generally more vulnerable to excitoxic effects of stress [14].

- $\quad$ GABA (inhibitory) activity is decreased, and glutamate (excitatory)activity is increased [14].

- Thyroid hormones become imbalanced leading to abnormal T3:T4 ratio and increases in anxiety [14].

- Increased dopamine and norepinephrine levels increase arousal, startle response, fear memory encoding and increased HPA-Axis activation in response to recapitulation [14].

- The volume of the hippocampus which controls not only the HPA-Axis and stress responses, but also declarative memory is reduced due to the excitotoxic environment [14].

- $\quad$ Sustained HPA-Axis activation causes persistently high levels of CRH which eventually causes a blunting of the ACTH response to CRH stimulation [14].

- Serotonin levels are simultaneously decreased in parts of the brain disrupting communication between the amygdala and the hippocampus which leads to increased vigilance, startle, impulsivity, and memory intrusions, hostility, aggression, depression, and suicidally [14].

- Amygdala activity increases and promotes hypervigilance and impairs threat discrimination [14].

- $\quad$ Reduced prefrontal cortex volume impairs executive functioning and impulse control [14].

- $\quad$ Reduced anterior cingulate volume impairs the extinction of fear responses [14].

- Changes to the ratios of estrogen, testosterone and progesterone occur which impact the body's ability to modulate cortisol levels [15]. For example, studies have shown that ovariectomies reduce and estrogen replacements increase the reactivity of the HPA axis. The hypothalamic-pituitary-gonadal (HPG) and HPA axes work together to maintain species survival and minimize allostatic load [16]. Other studies have shown that prolonged psychological stress suppresses estrogen causing amenorrhea which has profound effects on cardiac, skeletal, psychological and reproductive systems [17].

In addition to the physiological changes that occur in response to gluticocorticoid resistance, people in a state of hypocortisolism at the time of exposure to additional stressors develop stronger trauma-related symptoms in part due to the exaggerated HPA-Axis response causing the stressor to have a stronger negative emotional valence [14]. People become stuck in this loop in which their basal cortisol and energy levels are low most of the time, yet when there is a stressor, the body's response is highly exaggerated which taxes the system and cause the body to stay in a state of hypocortisolism. Due to the heightened emotional valence of negative experiences and information and encoding of these memories, even exposure to positive experiences at a 1:1 ratio to negative ones does not "balance" the stress response.

\section{Lifestyle Factors}

It isn't just traumatic stress that activates the HPA-Axis though. When the body perceives a physiological, social, environmental threat including lack of quality sleep, excessive noise, poor nutrition, exposure to stimulants like caffeine, social stress, and hormone and neurohormone imbalances which can cause or result from chronic activation of the HPA-Axis, it triggers the HPAAxis and the corresponding cascade of physiological changes [15]. Identification and modification of these so-called lifestyle factors can reduce chronic HPA-Axis activation and help prevent a myriad of chronic conditions. Some of the most pervasive stressors include the media, social media, sleep deprivation, sedentariness, obesity and poor nutrition. Since many mental illnesses have their onset in adolescence and early adulthood, possibly triggered by stress exposure in a vulnerable, developing brain, prevention is of the utmost importance [18].

\section{Media}

According to the social signal transduction theory of depression, perception of social threat by exposure social, symbolic, or imagined threats and adversity up-regulate the HPAAxis. Modern media recasts social, cultural and political events and highlights our current vulnerabilities to terrorism and dystopia 24 hours a day [19]. The persistence of these messages within this climate of heightened awareness and vigilance about domestic and international terrorism, causes chronic HPA-Axis activation which leads to the release of proinflammatory cytokines that can trigger depressed mood, anhedonia, fatigue, psychomotor retardation, and behavioral withdrawal [6].

These messages are of increased concern regarding youth who, depending on their developmental level, may not be able to discern something that is being recast from something that is still occurring, setting the stage for generalized anxiety to develop [19]. The excitoxic effects from frequent or persistent activation of the HPA-Axis in children is also of great concern, because, youth and adolescence is a time of rapid brain development making the brain more susceptible to injury. It is important to note that all people, not just adolescents, have a tendency to pay greater attention to and engage in more detailed cognitive processing of negative than positive information [20]. Therefore, exposure to the predominantly negative stories in the news results in increased negative emotional responses increasing HPA-Axis activation and anxiety-related behaviors [21]. 


\section{Social Media}

Social media is another example of a modern lifestyle factor that exposes people to social and symbolic threats and adversity. Social media has been defined as computer-mediated technology that allows one to create and share information and other forms of expression though virtual communities. In 2016, 98\% of young adults used approximately 7.6 different social media regularly [22]. Individuals who spent more than 120 minutes on social media per day or who visited social media sites more than 9 times per day had significantly increased odds of depression [23,24]. Increased time online is associated with decline in communication with family members, a reduction of the internet user's social circle, a reduction in sleep and increased feelings of depression and loneliness [25]. All of these behaviors and symptoms are triggers for and can be symptoms of HPA-Axis dysregulation [26].

Another social stressor that is all too prevalent on social media is people who need to experience approval and reassurance through their social media connections "liking" their posts. When this does not happen, people who are more invested in social media often experience HPA-Axis activation as evidenced by poorer sleep quality, lower self-esteem and increased anxiety and depression. Stronger associations between technology-based SCFS and depressive symptoms for unpopular individuals $[27,28]$.

Even people who are not seeking approval and validation can experience negative effects of social media. It is estimated that more than $50 \%$ of people have witnessed online hate in their "feeds" or in comments left on their posts. Approximately $10 \%$ have perpetrated online hate and $23 \%$ of people have been victimized by online hate [29].

Another way social media increases stress among young adults and adolescents is Fear Of Missing Out (FOMO). FOMO drives people to regularly check their social media feed to ensure they are not missing anything. This constant preoccupation with checking social media is associated with reduced work productivity, reduced involvement in real-life activities and relationships and higher anxiety when not online [30].

Even though social relationships are one of the greatest buffers against stress, social media relationships often do not provide the same benefits as real-life relationships [31]. Instead of enhancing social relationships, social media communication may lead to the mistaken impressions about physical appearance, success and happiness of other people, thus increasing feelings of jealousy (social threat) and depression [30,32,24]. Additionally, there is a strong positive correlation between amount of social media usage and perceptions of isolation [33]. Without a feeling of belonging from social relationships to help buffer against stress, people experience more distress and resultant HPA-Axis activation $[34,35]$.

\section{Sleep}

Sleep, in particular lack of quality sleep, is another aspect of modern lifestyles which contributes to HPA-Axis activation and development of chronic illnesses. Deep sleep has an inhibiting influence on the HPA axis, and activation of the HPA axis can lead to insomnia and 24 hour increases of ACTH and cortisol secretion [36] Likewise, sleep disruption or deprivation can lead to significant increases of plasma cortisol levels, reduction in serotonin and melatonin and increases in norepinephrine which further impair the quality of sleep and lead to hyperactivation of the HPA-Axis [3638].

According to the CDC, 1 in 3 adults does not get enough sleep [39]. There are many causes of sleep deprivation in American culture. Poor sleep hygiene including noisy sleep environments and blue light exposure; use of nicotine, alcohol of caffeine too close to bedtime and dependence upon sleep aids are among the most common.

\section{Noise Related Sleep Disturbances}

More than daytime noise, nighttime noise exposure causes more frequent awakening, less deep sleep and increased subjective disturbance and is correlated with an increased risk of HPA-Axis activation, cardiovascular disease, depression, anxiety. In fact, long-term nocturnal noise exposure $>42$ decibles is associated with a $14 \%$ increase in prescriptions for sleep medication and a $17 \%$ increase in risk for being on antidepressant or anti-anxiety medications $[40,41]$.

\section{Nutrition Related Sleep Disturbances}

A recent study of the 2007-2008 National Health and Nutrition Examination Survey (NHANES) found inadequate intake of vitamin A, calcium, selenium, carbohydrates, vitamin D, and lycopene to be associated with "poor sleep" and low levels of zinc and magnesium are implicated in the development of depression through overactivity of the HPA-Axis $[42,43]$. A significant negative correlation was found between sleep quality and low quality carbohydrate intake from processed foods [44].

Additionally, skipping breakfast and eating irregularly were strongly associated with hypoglycemia which can cause chronic HPA-Axis activation and poor sleep quality $[44,45]$.

\section{Environmentally Related Sleep Disturbances}

Another factor impacting sleep is working in buildings with lack of access to natural light, shift work and overnight work which prohibits the body from receiving cues from the environment which would regulate a 24-hour circadian rhythm. Nearly $20 \%$ of Americans are at risk for "graveyard shift work disorder" which is characterized by insomnia and daytime drowsiness. Insomnia at night causes people to experience frustration and increases stress because the person was drowsy all day and desperately wants to sleep but cannot. Daytime drowsiness also causes people to use stimulants to wake up or get energy throughout the day contributing to even more HPA-Axis activation [46].

The ubiquitous presence of blue light from digital devices and televisions is yet another modifiable lifestyle factor. Blue light disrupts the signals to the brain that trigger the production of melatonin. While this may not lead to as much frustration as 
insomnia, the reduction in duration of quality sleep will also trigger the stress response [36].

\section{Snoring and Apnea Related Sleep Disturbances}

According to the American Academy of sleep medicine, 26\% of adults have sleep apnea which is associated with HPA axis activation [47]. While there are not a lot of ways to prevent sleep apnea, use of a CPAP device has been shown to reduce HPA-Axis activation via reduced cortisol levels [48].

\section{Alcohol Related Sleep Disturbances}

According to the Centers for Disease Control (CDC), heavy drinking is defined as drinking more than 15 drinks per week for men and more than 8 drinks a week for women (CDC, 2018). Twenty percent (20\%) of Americans are heavy drinkers and 50\% of heavy drinkers drink more than 10 alcoholic beverages each day [49]. Alcohol stimulates the hypothalamic-pituitary-adrenal (HPA) axis, via the hypothalamus, and repeated alcohol exposure leads to a blunted HPA-Axis response which is associated with depressive symptoms such as anhedonia, fatigue and behavioral withdrawal as well as widespread inflammation and increases the risk for development of other chronic health problems [50].

Many people use alcohol specifically to help them "wind down" so they can get to sleep. While it is true that alcohol decreases the time it takes for people to fall asleep (sleep latency), and increases the quality and quantity of NREM sleep during the first half of the night, during the second half of the night sleep, as the depressant effects of the alcohol wears off, sleep becomes disrupted [51]. Within the USA, it is estimated that societal costs of alcohol-related sleep disorders exceeds $\$ 18$ billion [51,52].

\section{Nicotine Related Sleep Disturbances}

Nicotine use is pervasive in the United States. Recently with the introduction of e-cigarettes, more and younger people have begun using nicotine products [53]. The blunting of the HPA-Axis response in the face of persistent exposure to a stimulant (nicotine) is evident in the findings that recent nicotine use and lower dependence is associated with increased activation of the HPA-Axis, but as dependence goes up, response of the HPA-Axis decreases [54]. Research has also found a significant reciprocal, relationships between smoking and sleep disturbances. The stimulant effects of nicotine may intensify sleep problems and be used during waking hours to counteract the effects of sleep problems on cognitive function $[55,56]$.

\section{Caffeine Related Sleep Disturbances}

Caffeine is found not only in coffee, but also soda, chocolate, over the counter migraine medications, decongestants and some diet and workout supplements. Like other stimulants, caffeine has been found to cause a significant increase in cortisol levels. Interestingly, when caffeine was paired with a mental or physical stressor, cortisol and adrenaline levels exceeded levels seen when caffeine or stressors were encountered independently $[57,58]$.

\section{Malnutrition}

We already discussed the impact of nutritional deficiencies, eating low quality carbohydrates, skipping breakfast and erratic eating patterns on sleep quality and resultant HPA-Axis activation. However, nutritional deficits are implicated in a host of other problems that can dysregulate the HPA-Axis. Up to 95\% of some neurotransmitters are made in the gut [59]. Recently scientists have discovered the gut-brain axis which is a bi-directional system between the brain and gastrointestinal tract, that links emotional and cognitive centers of the brain with the digestive tract via the vagus nerve [60]. The influence of gut bacteria on behavior is becoming increasingly understood, introducing the possibility that alterations in gut bacteria may be important in the development of disorders of the nervous system including HPA-Axis dysregulation. A healthy gut microbiome has over 1000 species of bacteria and can decrease depression and anxiety, regulate sleep, appetite and improve cognition [61]. An unhealthy gut microbiome contributes to an exaggerated HPA-Axis response [61,62]. Additionally, a healthy diet that supports the creation of hormones and neurotransmitters will help the body regulate the balance between excitatory (e.g., norepinephrine, dopamine, and glutamate) and inhibitory (e.g., serotonin and GABA) neurotransmitters and positively affect neurological, emotional and behavioral responses [63]. Many of the hormones and neurotransmitters in the body are constructed from proteins (amino acids) with the help of carbohydrates, vitamins and minerals. Without adequate intake of these nutrients, the body cannot create norepinephrine, corticotropin releasing factor (CRH), serotonin, glutamate, dopamine and many more.

Serotonin is an inhibitory neurotransmitter which helps stabilize mood, downregulate the HPA-Axis and is broken down to create melatonin to promote sleep. It is constructed from the amino acid tryptophan with the help of iron, magnesium, vitamin B6, folic acid, vitamin C and zinc. Insufficient levels of any of these nutrients can lead to serotonin depletion and hamper the body's ability to down-regulate the HPA-Axis. Additionally, frequent intake of caffeine or other stimulants can cause serotonin levels to become depleted [64]. Another neurochemical, GABA is also an inhibitory neurotransmitter which is constructed from the amino acid glutamine with the help of vitamin B6. When either of these two nutrients is insufficient, the HPA-Axis will stay activated until sustained activation triggers the conservation response of hypocortisolism.

\section{Sedentariness}

Americans are becoming increasingly sedentary [65]. As stress levels increase, it is important to find ways to reduce related inflammation and oxidative stress from HPA-Axis activation to prevent chronic conditions. Exercise has been shown to moderate both inflammatory cytokines and oxidative stress [66]. Low intensity exercise (at 40\% VO2max) has even been shown to reduce cortisol levels and increase serotonin contributing to the relaxation response $[67,68]$. 
Additionally, research has demonstrated that unfit individuals have increased HPA, inflammatory, and cardiovascular reactivity indicating that individuals who maintain sedentary lifestyle may have slower recovery from acute stress [69-72]. These studies support the idea that exercise can reduce the consequences of HPA Axis activation [73-75].

\section{Summary}

While some stress is necessary for energy and motivation, and some stress in life is inevitable, frequent or persistent stress is toxic to our bodies producing a host of physiological changes that can cause chronic health problems including mood disorders, metabolic syndrome, diabetes, chronic pain, auto-immune disorders and hypothyroidism. These changes have a bidirectional influence on the HPA-Axis. Under conditions of stress, pain or inflammation, the HPA-Axis is activated. When the HPA-Axis is activated too much, it can produce inflammation, anxiety and depressive symptoms. Several factors that are common to the American lifestyle including being inundated with information about threats to our social welfare from the news media, excessive engagement with and exposure to disinhibition on social media, insufficient quality sleep, poor nutrition, use of alcohol, nicotine and caffeine and sedentariness significantly contribute to the persistent activation of the HPA-Axis. Each of these factors is completely modifiable and can reduce the demands on the HPA-Axis as well as ensure it has all of the building blocks it needs to function effectively.

\section{Acknowledgement}

None.

\section{Conflict of Interest}

No conflict of interest.

\section{References}

1. Anaissie J, DeLay KJ, Wang W, Hatzichristodoulou G, Hellstrom WJ (2017) Testosterone deficiency in adults and corresponding treatment patterns across the globe. Translational andrology and urology 6(2): 183-191.

2. Patel S, Homaei A, Raju AB, Meher BR (2018) Estrogen: The necessary evil for human health, and ways to tame it. Biomed Pharmacother 102: 403-411.

3. American Heart Association (2018) Retrieved 10/21/2019 from https://healthmetrics.heart.org/wp-content/uploads/2018/02/At-AGlance-Heart-Disease-and-Stroke-Statistics-2018.pdf

4. Moore JX, Chaudhary N, Akinyemiju T (2017) Metabolic Syndrome Prevalence by Race/Ethnicity and Sex in the United States, National Health and Nutrition Examination Survey, 1988-2012. Prev Chronic Dis 14: 160287.

5. Bullard KM, Cowie CC, Lessem SE, Saydah SH, Menke A, et al. (2018) Prevalence of Diagnosed Diabetes in Adults by Diabetes Type - United States, 2016. MMWR. Morbidity and mortality weekly report 67(12): 359-361.

6. Slavich GM, Irwin MR (2014) From stress to inflammation and major depressive disorder: a social signal transduction theory of depression. Psychological bulletin 140(3): 774-815.

7. Du X, Pang TY (2015) Is Dysregulation of the HPA-Axis a Core Pathophysiology Mediating Co-Morbid Depression in Neurodegenerative Diseases? Frontiers in psychiatry 6: 32.
8. Stephens MA, Wand G (2012) Stress and the HPA axis: role of glucocorticoids in alcohol dependence. Alcohol research : current reviews 34(4): 468-483.

9. Lin TK, Zhong, L, Santiago JL (2017) Association between Stress and the HPA Axis in the Atopic Dermatitis. International journal of molecular sciences 18(10): 2131.

10. Silverman MN, Pearce BD, Biron CA, Miller AH (2005) Immune modulation of the hypothalamic-pituitary-adrenal (HPA) axis during viral infection. Viral immunology 18(1): 41-78.

11. Ben-Zee, A (2010) Retrieved 10/21/2019 from https://www. psychologytoday.com/us/blog/in-the-name-love/201007/arenegative-emotions-more-important-positive-emotions

12. Bowen HJ, Kark SM, Kensinger EA (2018) NEVER forget: negative emotional valence enhances recapitulation. Psychonomic bulletin \& review 25(3): 870-891.

13. Spencer RL, Deak T (2017) A users guide to HPA axis research. Physiology \& behavior. Psychoneuroendocrinology 178: 43-65.

14. Sherin JE, Nemeroff CB (2011) Post-traumatic stress disorder: the neurobiological impact of psychological trauma. Dialogues in clinical neuroscience 13(3): 263-278.

15. Joseph D N, Whirledge S (2017) Stress and the HPA Axis: Balancing Homeostasis and Fertility. International journal of molecular sciences 18(10): 2224

16. Oyola MG, Handa RJ (2017) Hypothalamic-pituitary-adrenal and hypothalamic-pituitary-gonadal axes: sex differences in regulation of stress responsivity. Stress (Amsterdam, Netherlands): 20(5): 476-494.

17. Shufelt CL, Torbati T, Dutra E (2017) Hypothalamic Amenorrhea and the Long-Term Health Consequences. Seminars in reproductive medicine 35(3): 256-262.

18. Furhmann D, Knoll LJ, Blakemore S (2015) Adolescence as a Sensitive Period of Brain Development Trends in Cognitive Sciences. Trends Cogn Sci 19(10): 558-566.

19. Comer JS, Furr JM, Beidas RS, Weiner CL, Kendall PC (2008) Children and terrorism-related news: training parents in Coping and Media Literacy. Journal of consulting and clinical psychology 76(4): 568-578.

20. Kätsyri J, Kinnunen T, Kusumoto K, Oittinen P, Ravaja N (2016) Negativity Bias in Media Multitasking: The Effects of Negative Social Media Messages on Attention to Television News Broadcasts. PloS one 11(5): e0153712.

21. Kleemans M, Schlindwein L F, Dohmen R (2017) Preadolescents' Emotional and Prosocial Responses to Negative TV News: Investigating the Beneficial Effects of Constructive Reporting and Peer Discussion. Journal of youth and adolescence 46(9): 2060-2072.

22. Villanti AC, Johnson AL, Ilakkuvan V, Jacobs MA, Graham AL, et al. (2017) Social Media Use and Access to Digital Technology in US Young Adults in 2016. Journal of medical Internet research 19(6): e196.

23. Lin LY, Sidani JE, Shensa A, Radovic A, Miller E, et al. (2016) Association Between Social Media Use and Depression Among U.S. Young Adults. Depression and Anxiety 33(4): 323-331.

24. Shensa A, Sidani JE, Dew MA, Escobar-Viera CG, Primack BA (2018) Social Media Use and Depression and Anxiety Symptoms: A Cluster Analysis. American journal of health behavior 42(2): 116-128.

25. Pantic I, Damjanovic A, Todorovic J, Topalovic D, Bojovic-Jovic D, et al. (2012) Association between online social networking and depression in high school students: behavioral physiology viewpoint. Psychiatr Danub 24(1): 90-93.

26. Kraut R, Patterson M, Lundmark V, Kiesler S, Mukopadhyay T, et al (1998) Internet paradox. A social technology that reduces social involvement and psychological well-being? The American Psychologist 53: 10171031

27. Nesi J, Prinstein M J (2015) Using Social Media for Social Comparison and Feedback-Seeking: Gender and Popularity Moderate Associations 
with Depressive Symptoms. Journal of abnormal child psychology 43(8): 1427-1438.

28. Woods HC, Scott H (2016) \#Sleepyteens: Social media use in adolescence is associated with poor sleep quality, anxiety, depression and low selfesteem. J Adolesc 51: 41-49.

29. Wachs S, Wright MF (2018) Associations between Bystanders and Perpetrators of Online Hate: The Moderating Role of Toxic Online Disinhibition. International journal of environmental research and public health 15(9): 2030.

30. Pantic I (2014) Online social networking and mental health. Cyberpsychology, behavior and social networking, 17(10): 652-657.

31. Van Schalkwijk FJ, Blessinga AN, Willemen AM, Van Der Werf YD, Schuengel C (2015) Social support moderates the effects of stress on sleep in adolescents. Journal of Sleep Research 24(4): 407-413.

32. Chou HT, Edge N (2012) "They are happier and having better lives than I am": The impact of using Facebook on perceptions of others' lives. Cyberpsychology, Behavior \& Social Networking 15: 117-121.

33. Primack BA, Shensa A, Sidani JE, Whaite EO, Lin LY, et al. (2017) Social Media Use and Perceived Social Isolation Among Young Adults in the US. American journal of preventive medicine 53(1): 1-8.

34. Butler TR, Karkhanis AN, Jones SR, \& Weiner JL (2016) Adolescent Social Isolation as a Model of Heightened Vulnerability to Comorbid Alcoholism and Anxiety Disorders. Alcoholism, clinical and experimental research 40(6): 1202-1214.

35. Taylor HO, Taylor RJ, Nguyen AW, Chatters L (2018) Social Isolation, Depression, and Psychological Distress Among Older Adults. Journal of aging and health 30(2): 229-246.

36. Chrousos G, Vgontzas AN, Kritikou I (2016) HPA Axis and Sleep. In: Feingold KR, Anawalt B, Boyce A, et al, (Eds.), Endotext [Internet]. South Dartmouth (MA): MDText.com, Inc.; 2000, USA.

37. Meerlo P, Sgoifo A, Suchecki D (2008) Restricted and disrupted sleep: effects on autonomic function, neuroendocrine stress systems and stress responsivity. Sleep Med Rev 12(3): 197-210.

38. Balbo, M, Leproult, R, Van Cauter, E (2010) Impact of Sleep and Its Disturbances on Hypothalamo-Pituitary-Adrenal Axis Activity. International Journal of Endocrinology 2010: 16.

39. Centers for Disease Control (2016) Retrieved 10/21/2019 from https:// www.cdc.gov/media/releases/2016/p0215-enough-sleep.html

40. Poulsen AH, Raaschou-Nielsen O, Peña A, Hahmann AN, Nordsborg RB et al. (2019) Impact of Long-Term Exposure to Wind Turbine Noise on Redemption of Sleep Medication and Antidepressants: A Nationwide Cohort Study. Environmental health perspectives 127(3): 37005.

41. Beutel ME, Jünger C, Klein EM, Wild P, Lackner K, et al. (2016) Noise Annoyance Is Associated with Depression and Anxiety in the General Population- The Contribution of Aircraft Noise. PloS one 11(5): e0155357.

42. Wang J, Um P, Dickerman BA, Liu J (2018) Zinc, Magnesium, Selenium and Depression: A Review of the Evidence, Potential Mechanisms and Implications. Nutrients 10(5): 584

43. Grandner MA, Alfonso-Miller P, Fernandez-Mendoza J, Shetty S, Shenoy S, et al. (2016) Sleep: important considerations for the prevention of cardiovascular disease. Current opinion in cardiology 31(5): 551-565.

44. St-Onge MP, Mikic A, Pietrolungo CE (2016) Effects of Diet on Sleep Quality. Advances in nutrition 7(5): 938-949.

45. Harrell CS, Gillespie CF, Neigh GN (2016) Energetic stress: The reciprocal relationship between energy availability and the stress response. Physiology \& behavior 166: 43-55.

46. Salgado-Delgado R, Tapia Osorio A, Saderi N, Escobar C (2011) Disruption of circadian rhythms: a crucial factor in the etiology of depression. Depression research and treatment: 839743.

47. Kritikou I, Basta M, Vgontzas AN, Pejovic S, Fernandez-Mendoza J, et al. (2016) Sleep apnoea and the hypothalamic-pituitary-adrenal axis in men and women: effects of continuous positive airway pressure. European Respiratory Journal 47: 531-540.

48. Tasali E, Chapotot F, Leproult R, Whitmore H, Ehrmann DA (2011) Treatment of obstructive sleep apnea improves cardiometabolic function in young obese women with polycystic ovary syndrome. The Journal of clinical endocrinology and metabolism 96(2): 365-374.

49. Ingraham C (2014) The Washington Post https://www.washingtonpost. com/news/wonk/wp/2014/09/25/think-you-drink-a-lot-this-chartwill-tell-you/

50. Allen CD, Lee S, Koob GF, Rivier C (2011) Immediate and prolonged effects of alcohol exposure on the activity of the hypothalamic-pituitaryadrenal axis in adult and adolescent rats. Brain, behavior, and immunity 25 Suppl 1(Suppl 1): S50-S60.

51. Thakkar MM, Sharma R, Sahota P (2015) Alcohol disrupts sleep homeostasis. Alcohol 49(4): 299-310.

52. Devenney LE, Coyle KB, Roth T, Verster JC (2019) Sleep after Heavy Alcohol Consumption and Physical Activity Levels during Alcohol Hangover. Journal of clinical medicine 8(5): 752.

53. National Institute of Diabetes and Digestive and Kidney Diseases (2019) from https://www.niddk.nih.gov/health-information/endocrinediseases/hypothyroidism\#common

54. Morris MC, Mielock AS, Rao U (2016) Salivary stress biomarkers of recent nicotine use and dependence. The American journal of drug and alcohol abuse 42(6): 640-648.

55. Davila DG, Hurt RD, Offord KP, Harris CD, Shepard JW (1994) Acute effects of transdermal nicotine on sleep architecture, snoring, and sleepdisordered breathing in nonsmokers. Am J Respir Crit Care Med 150(2): 469-474.

56. Bellatorre A, Choi K, Lewin D, Haynie D, Simons-Morton B (2017) Relationships Between Smoking and Sleep Problems in Black and White Adolescents. Sleep 40(1): zsw031.

57. Lovallo WR, Farag NH, Vincent AS, Thomas TL, Wilson MF (2006) Cortisol responses to mental stress, exercise, and meals following caffeine intake in men and women. Pharmacology, biochemistry, and behavior, 83(3): 441-447.

58. Graham TE, Hibbert E, Sathasivam P (1998) Metabolic and exercise endurance effects of coffee and caffeine ingestion. Metabolism: 883-889.

59. Mirza S (2015) Neurotransmitters: the link between depression, chronic fatigue and chronic pain syndromes. BMJ 2015350

60. Zhu X, Han Y, Du J, Liu R, Jin K, et al. (2017) Microbiota-gut-brain axis and the central nervous system. Oncotarget 8(32): 53829-53838.

61. Liu L, Zhu G (2018) Gut-Brain Axis and Mood Disorder. Frontiers in psychiatry 9: 223

62. Jenkins TA, Nguyen JC, Polglaze KE, Bertrand PP (2016) Influence of Tryptophan and Serotonin on Mood and Cognition with a Possible Role of the Gut-Brain Axis. Nutrients 8(1): 56.

63. Armborst D, Metzner C, Alteheld B, Bitterlich N, Rösler D, et al. (2018) Impact of a Specific Amino Acid Composition with Micronutrients on Well-Being in Subjects with Chronic Psychological Stress and Exhaustion Conditions: A Pilot Study. Nutrients 10(5): 551.

64. Shi D, Nikodijević O, Jacobson KA, Daly JW (1993) Chronic caffeine alters the density of adenosine, adrenergic, cholinergic, GABA, and serotonin receptors and calcium channels in mouse brain. Cellular and Molecular Neurobiology. 13(3): 247-261.

65. National Institute of Health (2008) Children's Physical Activity Drops From Age 9 to 15, NIH Study Indicates. Retrieved 10/21/2019 from https://www.nih.gov/news-events/news-releases/childrens-physicalactivity-drops-age-9-15-nih-study-indicates.

66. Sarris J, Neil A, Coulson CE, Schweitzer I, Bberk M (2014) Lifestyle Medicine for Depression. BMC Psychiatry 14: 107.

67. Young SN (2007) How to increase serotonin in the human brain without drugs. Journal of psychiatry \& neuroscience: JPN, 32(6): 394-399. 
68. Hill EE, Zack E, Battaglini C, Viru A, Hackney AC (2008) Exercise and circulating cortisol levels: the intensity threshold effect. J Endocrinol Invest 31(7): 587-591.

69. Puterman E, Donovan A, Adler NE, Tomiyama AJ, Kemeny M, et al. (2011) Physical activity moderates effects of stressor-induced rumination on cortisol reactivity. Psychosomatic medicine 73(7): 604-611.

70. American Autoimmune Related Diseases Association (2019) Retrieved 10/22/2019 from https://www.aarda.org/news-information/ statistics/

71. Anxiety and Depression Association of America(2019) from https:// adaa.org/about-adaa/press-room/facts-statistics
72. Centers for Disease Control (2018) Retrieved 10/21/2019 from https:// www.cdc.gov/alcohol/faqs.htm\#heavyDrinking

73. Division of Population Health, National Center for Chronic Disease Prevention and Health Promotion, Centers for Disease Control and Prevention Retrieved March 29, 2018 from https://www.cdc.gov/ alcohol/faqs.htm\#heavyDrinking

74. National Center for Chronic Disease Prevention and Health Promotion (NCCDPHP) Retrieved 10/21/2019 from https://www.cdc.gov/ chronicdisease/about/costs/index.htm

75. National Institutes of Health (2019) Vaping Rises Among Teens, USA. 